Research Paper

\title{
Trisenox Disrupts MDM2-DAXX-HAUSP Complex and Induces Apoptosis in a Mouse Model of Acute Leukemia
}

\author{
Sanjay Kumar and Paul B. Tchounwou ${ }^{凶}$ \\ Cellomics and Toxicogenomics Research Laboratory, NIH/NIMHD-RCMI Center for Environmental Health, College of Science, Engineering and Technology, \\ Jackson State University, 1400 Lynch Street, Box18750, Jackson, Mississippi, MS 39217, USA. \\ $\square$ Corresponding author: Paul B. Tchounwou; Cellomics and Toxicogenomics Research Laboratory, NIH/NIMHD-RCMI Center for Environmental Health, \\ College of Science, Engineering and Technology, Jackson State University, 1400 Lynch Street, Box18750, Jackson, Mississippi, MS 39217, USA; Tel: (601) 979-0777; \\ Fax: (601) 979-0570; Email: paul.b.tchounwou@jsums.edu \\ (1) The author(s). This is an open access article distributed under the terms of the Creative Commons Attribution License (https://creativecommons.org/licenses/by/4.0/). \\ See http://ivyspring.com/terms for full terms and conditions.
}

Received: 2019.09.04; Accepted: 2020.01.12; Published: 2020.05.12

\begin{abstract}
Trisenox (TX) is successfully used for both de novo and relapsed acute promyelocytic leukemia (APL) treatment. Although TX toxicity to APL cells is mediated by oxidative stress, DNA damage, cell cycle arrest, and apoptosis, its mode of action in the transgenic mice model of APL is poorly understood. We hypothesized that TX regulates cell cycle and apoptosis in APL mice by $\mathrm{p} 53$ activation, DNA damage, and reduced expression of MDM2-DAXX-HAUSP complex. To test hypothesis, we treated APL mice with different doses $(0,1.25 .2 .5 .5 .0 \& 7.5 \mathrm{mg} / \mathrm{kg}$ body $\mathrm{wt})$ of $\mathrm{TX}$ and collected the liver and bone marrow cells. We applied several techniques to check the expression of PML-RAR $\alpha$, complex molecules, and DNA damage in APL mice bone marrow cells and liver. Our findings indicate that TX reduced the expression of PML-RAR $\alpha$ and complex molecules, induced DNA damage and activated p53 leading to cell cycle arrest and apoptosis in APL mice liver. We found that TX promoted more promyelocytes formation with dense granules in bone marrow cells. It also transmitted the DNA damage signal through protein kinase (ATM \& ATR) leading to disruption of complex and activation of p53 in APL mice liver. TX induced cell cycle arrest through activation of $\mathrm{p} 53, \mathrm{p} 21$, and reduced expression of cyclin D1 and cyclin dependent kinases (CDK 2, 4 \& 6) in mice liver. It also caused apoptosis through upregulation of caspase 3 and Bax expression, and down-regulation of $\mathrm{Bcl} 2$ expression. Taken together, these molecular targets provide new insights into TX mode of action in APL mice.
\end{abstract}

Key words: Trisenox, p53, DAXX, HAUSP, MDM2.

\section{Introduction}

APL, a subtype of acute myeloid leukemia (AML) that is formed inside the bone marrow through a translocation mutation between chromosome 15 and chromosome 17, affects about 1,500 people in the United States annually $(1,2)$. It results from the formation of two fusion genes (oncogenes); promyelocytic leukemia-retinoic acid receptor alpha (PML-RARa) and RARa-PML. PML-RARa fusion transcript is involved in the pathogenesis of APL whereas RARa-PML fusion transcript is an important molecular marker for the diagnosis and monitoring of APL $(2,3)$. TX has been used successfully for treatment of all age groups of APL patients in both induction and consolidation therapy either alone or in combination with all trans retinoic acid (ATRA) with a complete remission and high survival rate $(2,4)$. Recently, few TX resistant APL patients have been reported in different parts of the world with X- RARa oncogenes $(5,6)$.

$\mathrm{P} 53$, is a tumor suppressor protein $(7,8)$, induces cell cycle arrest and apoptosis in response of DNA damage and other stresses in several cancer cells $(9,10)$. Its expression level is kept low in normal/unstressed cells by several ubiquitin ligases (E3), predominantly mouse double minute 2(Mdm2) and their isoform Mdm4/MDMX, through proteasomal degradation and ubiquitination (11). MDM2 is an unstable protein and also a negative 
regulator of p53 that remains associated with DAXX, and HAUSP in the form of tertiary complex (12). This complex reduces self-ubiquitination of MDM2, maintaining MDM2 ligase activity toward p53 in normal living cells. Exposure of cells to genotoxic stress [DNA damage], oxidative stress, hypoxia, and heat shock leads to MDM2-DAXX-HAUSP complex disruption, and stimulation of MDM2 self-ubiquitination and degradation leading to accumulation of p53 $(7,12-16)$. Scientific evidence suggests that TX stimulates p53 by DNA damage, $\mathrm{p} 21$ induction, cell cycle arrest at G1 phase, and apoptosis in fibroblast cells (17), human gastric cancer cells (18) and in HL-60 cells (8). It has been reported that TX inhibits cell proliferation with interaction with p21 leading to cell cycle arrest and apoptosis in human myeloma cells (19), HL-60 cells $(20,21)$, lymphoid malignant cells (22) and NB4 cells (23). TX stimulates accumulation of death domain-associated protein (DAXX), a nuclear protein that modulates transcription of death-related genes in apoptosis (24). Berberine has been reported to cause apoptosis by interaction of DAXX, disruption of MDM2-DAXX-HAUSP complex and degradation of MDM2 in acute lymphoblastic leukemia cells (12), while doxorubicin and VP-16 kill cancer cells through disruption of DAXX-MDM2-HAUSP complex, self-ubiquitination and degradation of MDM2, which leads to accumulation of p53 $(12,25)$. Promyelocytic leukemia (PML) gene interacts with p53 inside PML-nuclear body (NB), and is actively involved in p53 dependent pro-apoptotic events (26). Promyelocytic leukemia zinc finger-retinoic acid receptor a (PLZF-RARa), an oncogene transcriptional repressor, regulates cell proliferation in APL patients by dow-regulation of $\mathrm{p} 53$ and $\mathrm{p} 21$ proteins expression (27). Activation of PML-transformation related protein (Trp53) is necessary to control leukemiainitiating cells in mouse model of APL (28). Pseudokinase Tribble 3 (TRIB3) stimulates APL progression by inhibition of p53 mediated senescence and PML-RARa stabilization. RARa / arsenic trioxide interacts with TRIB3/ PML-RARa and eradicates APL by degradation of PML-RARa (29). In the present research, we revealed TX mode of action in a mice model of APL; involving DNA damage, stress signal transmission, reduced complex molecules expression, and activation of p53 leading to cell cycle regulation and apoptosis APL mice tissues. TX also promoted more promyelocytes formation with dense granules, and reduced PML-RARa expression in bone marrow cells. Understanding the molecular mechanism of TX action in mice model of APL would be very helpful for the design of new APL drugs.

\section{Material and Methods}

\section{Chemicals and Reagents}

Trisenox (arsenic trioxide) was purchased from Fisher scientific (Waltham, MA). Mitochondrial isolation kit, caspase assay kit, protease inhibitor, wright-giemsa solution and glutathione assay kit were obtained from Sigma-Aldrich (St. Louis, MO). Anti-cytochrome C, anti-Bax and anti-Bcl2 were purchased by Cell Signaling Technology (Danvers, MA). Caspase 3 activity assay kit was obtained from Abcam (Cambridge, MA). Hoechst 33342, Alexa fluor 568 and Alexa fluor 568 were purchased from Life Technologies (Grand Island, NY).

\section{Transgenic APL mice}

Acute promyelocytic leukemia (APL) transgenic mice were purchased from The Jackson Laboratory in Bar Harbor, Maine, USA. Our APL transgenic mice strain is C57BL/6-Pmltm1(PML/RARA)Ley/J and stock No: 017959 having gene construct, promyelocytic leukemia-retinoic acid receptor alpha (PML- RAR $\alpha$ ) widely expressed fusion proteins and tag with Cre recombinase. APL transgenic mice were kept in the Animal Core facility following the guidelines and recommendations of Jackson State University IACUC Committee. After one month of acclimatization, they were bred to produce enough mice for experimentation. Regular genotyping was done from young pups mice tail blood and proper homozygous mice containing our desired oncogene (PML-RAR $\alpha$ ) responsible for pathogenesis of APL were maintained $(29,30)$. Young transgenic mice (8-12 weeks old with average weight of 20-30g) were used for this experiment. Each treatment group was made of five young mice of similar weight and age. They were treated with different doses of $\operatorname{TX}(1.25,2.5$. 5.0 and $7.5 \mathrm{mg} / \mathrm{kg}$ body $\mathrm{wt}$ ) for 21 days by a continuous intraperitoneal injection based on previous publication (30) and our standardization procedure. After 21 days of TX treatment of transgenic mice, the liver tissue was dissected and the bone marrow cells were isolated for further experimentation.

\section{Bone marrow isolation from transgenic mice}

Young transgenic mice (8-12 weeks old with average weight of 20-30g) were treated with different doses of TX $(1.25,2.5 .5 .0$ and $7.5 \mathrm{mg} / \mathrm{kg}$ body wt) for 21 days continuously. After treatment, the transgenic mice were euthanized through $\mathrm{CO}_{2}$ asphyxiation, and bone marrow cells were isolated from the femur and tibia as described previously (31).

\section{Wright staining of promyelocytes inside the bone marrow cells}

We made thin smears of sterile bone marrow 
cells of all doses TX-treated or untreated mice samples on glass slides and promyelocytes were stained with Wright-Giemsa solution as previously described (32). In brief, air dry bone marrow cells smears were placed in Wright-Giemsa solution for about 3 minutes. After incubation, slides were dipped in phosphate buffer $(\mathrm{pH}=7.2)$ for 10 minutes. Then, slides were rinsed with distilled water, air dry, and the stained promyelocytes images were taken using Arcturus Laser Capture Micro dissection (LCM) System (Grand Island, NY).

\section{Immunocytochemistry and confocal microscopy imaging}

APL bone marrow cells $\left(1 \times 10^{5}\right)$ were cultured in presence or absence of TX and attached on poly-L-lysine coated slides. Immunocytochemistry of attached cells was performed using Ki67 antibody (dilution, 1:100) (cat\# 33-4711) or p53 antibody (cat \# 9282) and PML-RAR $\alpha$ (cat\# ab43152) from Life Technology, Cell Signaling or Abcam company and imaged by confocal microscopy (Olympus Company, Center valley, PA) as previously described (2).

\section{Tunnel Assay}

The TUNEL assay is very sensitive technique to analyze DNA damage in tissue sections. In brief, we treated transgenic APL mice with different TX doses $(0,1,2,4,6$ and $8 \mathrm{mg} / \mathrm{kg}$ body $\mathrm{wt})$ and collected the livers in RIPA buffer. Liver tissues were frozen in embedding medium( Polarstat Plus) and $5 \mu \mathrm{M}$ sections were made using Cryostar NX50 (Thermo Scientific, Waltham, MA) and DNA damage was analyzed by immunochemistry and confocal imaging using Promega DeadEnd ${ }^{\mathrm{TM}}$ Fluorometric TUNEL System Technical Bulletin (Cat\# G3250) or as previously described (33). Liver sections were fixed in acetone and methanol mixture at $-200 \mathrm{C}$ for $5 \mathrm{~min}$ and permeabilized with $0.2 \%$ triton $\mathrm{X}$ at $4{ }^{0} \mathrm{C}$ for $10 \mathrm{~min}$. Slides were washed with PBS two times and equilibrated in equilibration buffer for $10 \mathrm{~min}$ at room temperature. $100 \mu \mathrm{L}$ rTdT incubation buffer were added on each slide and incubated at $37^{0} \mathrm{C}$ for one hour. After incubation, the reaction was terminated by dipping slides in 2X SSC for $15 \mathrm{~min}$ and washed 2-3 times with PBS. The slides were stained with DAPI and mounted by anti-fade solution with coverslip and nail polish. After drying of slides, confocal imaging was performed using the Fluoview confocal microscopy system (Olympus company) (2).

\section{Immunoprecipitation and Western blotting}

After treatment of APL cells and APL mice with different doses of $\mathrm{TX}$, mice liver tissue and bone marrow cells were collected and protein lysates were prepared in RIPA buffer by sonication and centrifugation. We used $500 \mu \mathrm{g}$ protein lysate of liver tissues or APL cells of each sample and immunoprecipitation (IP) performed according to standard Thermofisher Scientific protocol and described earlier (12). For regular western blotting, an equal amount $(40 \mathrm{mg})$ of protein lysate from control or TX treated cells or tissues were loaded per lane on a $10 \%$ SDS-PAGE gel, transferred into a nitrocellulose membrane and analyzed by western blotting by using specific antibody as described previously (2). The band intensities were quantified using Image $\mathrm{J}$ software.

\section{Immunohistochemistry (IHC)}

We collected in RIPA buffer the livers of both control and APL mice treated with different doses (1, 2, 4, 6 and $8 \mathrm{mg} / \mathrm{kg}$ body wt) of TX for 21 days continuously intraperitonially. Liver tissues were frozen in embedding medium (Polarstat Plus), and $5 \mu \mathrm{M}$ sections were made using Cryostar NX50 ( Thermo Scientific, Waltham, MA). Liver sections were fixed in acetone and methanol mixture at $-20{ }^{0} \mathrm{C}$ for 5 min and permeabilized with $0.2 \%$ triton $\mathrm{X}$ at $4{ }^{0} \mathrm{C}$ for $10 \mathrm{~min}$. They were washed three times with PBS and blocked in 5\% normal goat serum containing $4 \%$ BSA for $30 \mathrm{~min}$ at room temperature. Blocked sections were incubated in anti-p53 (1:100) and anti-MDM2 (1:100) antibodies inside humidified chamber for four hours at room temperature. Again, the sections were washed three times with PBS and further incubated with secondary antibody [Alexa fluor 488 \& 594(1:1000)] for one hour at room temperature. After incubation, the sections were washed with PBS and the images were captured under fluorescence microscope, IX73 (Olympus, Center Valley, PA) and presented as shown previously (34).

\section{Statistical analysis}

Experiments were performed in triplicates. Data were presented as means \pm SDs. Where appropriate, one-way ANOVA or student paired t-test was performed using SAS Software available in the Biostatistics Core Laboratory at Jackson State University. P-values less than 0.05 were considered statistically significant.

\section{Results}

\section{TX stimulates more promyelocytes formation and reduces PML-RAR $\alpha$ expression}

To study TX effect on transgenic mouse model of APL, we treated mice with different doses $(0,1.25,2.5$, 5 and $7.5 \mathrm{mg} / \mathrm{kg}$ body weight) of TX and isolated bone marrow cells. We made thin smears of bone marrow cells on slides, dry, fixed with phosphate 
buffer, stained with Wright-Giemsa staining, and imaged by fluorescence microscopy. Our results show that TX stimulated more promyelocytes formation with dense granules in treated APL mice bone marrow cells in a dose-dependent manner (Fig.1A). Our immunocytochemistry and confocal imaging findings show that TX also reduced the expression level of PML-RARa oncogene in bone marrow cells, in a dose-dependent manner (Fig.1B[i-v]).

\section{TX induces DNA damage in APL mice liver}

To investigate the genotoxic effect of TX on APL mice liver, we collected liver tissues and made $5 \mu \mathrm{m}$ sections after treatment with different doses of TX. We then performed immunohistochemistry and tunnel assay to assess TX-induced DNA damage in APL mice liver tissue. Our findings show that TX induced DNA damage in APL liver tissue in a dose-dependent fashion $(2[i-v])$.
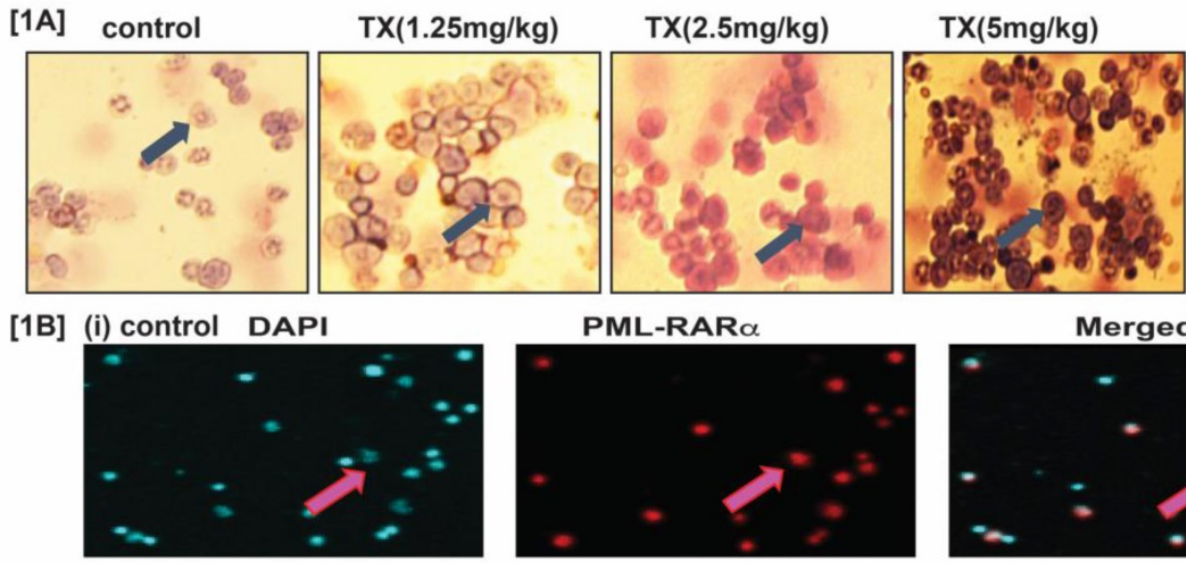

$\mathrm{TX}(7.5 \mathrm{mg} / \mathrm{kg})$

(ii) $\mathrm{TX}(1.25 \mathrm{mg} / \mathrm{kg}$ body wt)
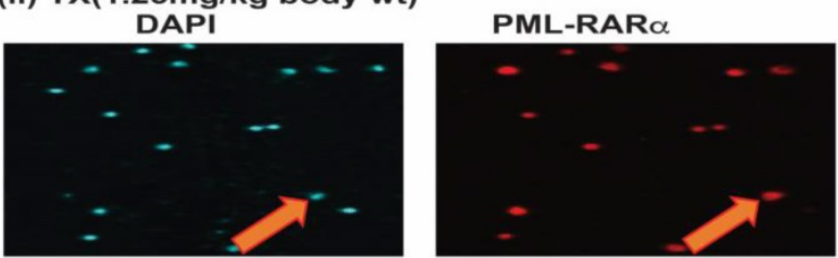

(iii) $\mathrm{TX}(2.5 \mathrm{mg} / \mathrm{kg}$ body wt) DAPI

PML-RARa

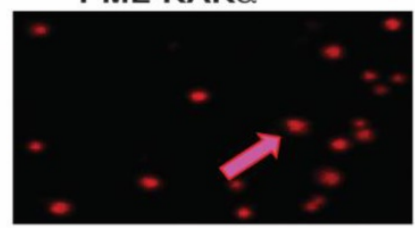

PML-RAR $\alpha$

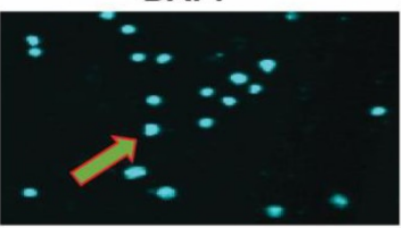

(iv TX(5mg/kg body wt)

DAPI

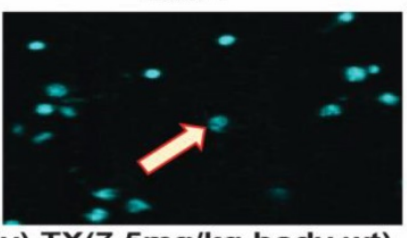

(v) TX(7.5mg/kg body wt) DAPI

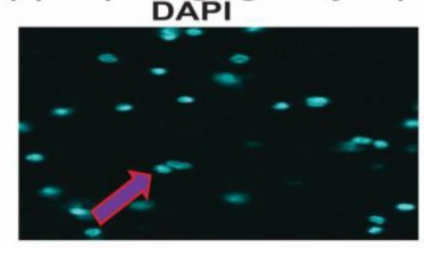

PML-RARa

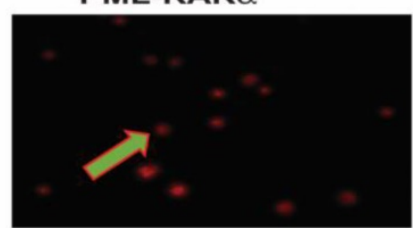

PML-RAR $\alpha$

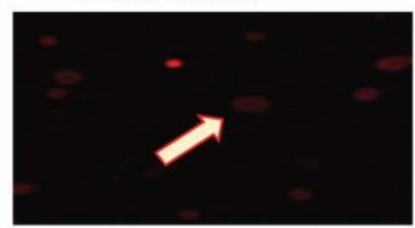

PML-RAR $\alpha$

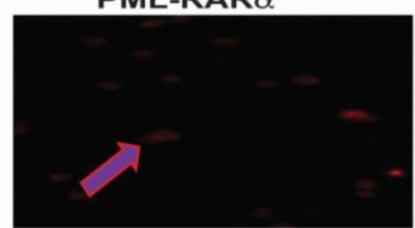

Merged
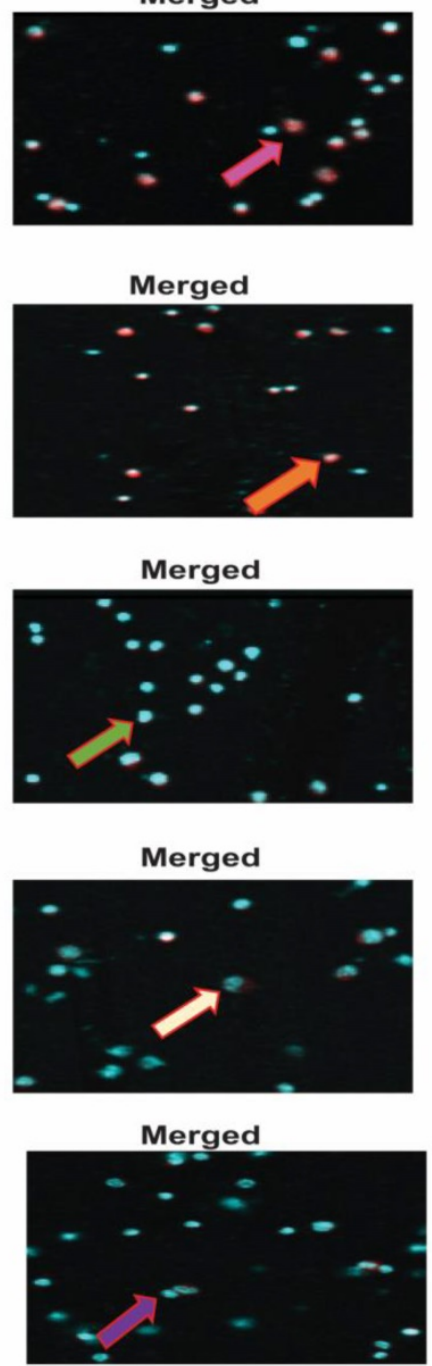

Figure 1. TX stimulates more promyelocytes formation and reduces PML-RAR $\alpha$ expression. APL transgenic mice were treated intraperitoneally with different doses $(0,1.25,2.5,5.0 \& 7.5 \mathrm{mg} / \mathrm{kg}$ body wt) of TX for 21 days. After treatment, the mice were sacrificed and the bone marrow cells were collected. Promyelocytes were stained with Wright staining and PML-RAR $\alpha$ expression analyzed by immunocytochemistry and confocal imaging in APL bone marrow cells. TX promoted more promyelocytes with dense granules formation and reduced PML-RARa expression in APL mice bone marrow cells (Fig. 1A \&B [i-v]). 


\section{[2](i) Control}

Hoechst 33342

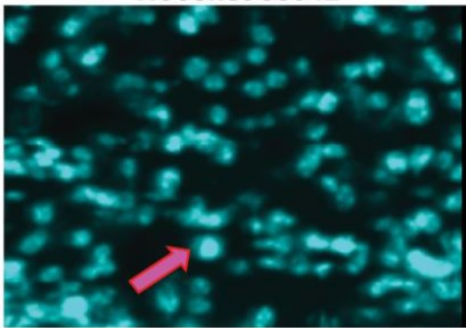

(ii)TX(1.25 mg/kg body wt) Hoechst 33342

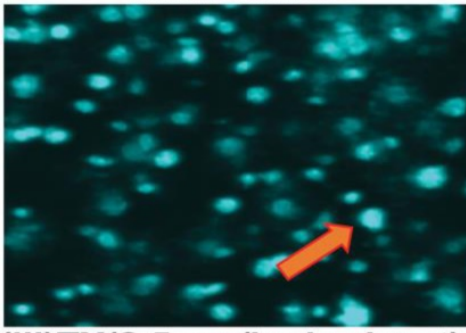

(iii)TX(2.5 mg/kg body wt)

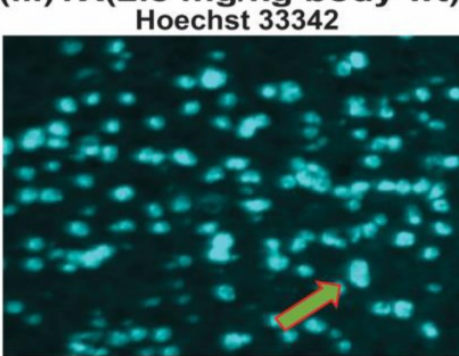

(iv)TX( $5 \mathrm{mg} / \mathrm{kg}$ body wt)

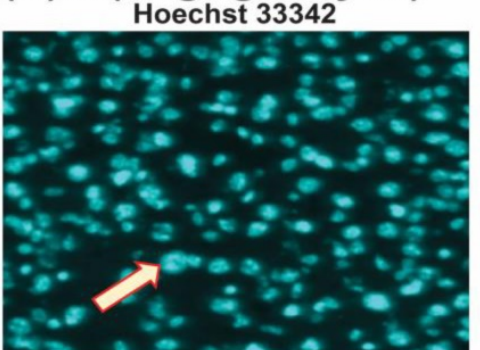

(v)TX(7.5mg/kg body wt)

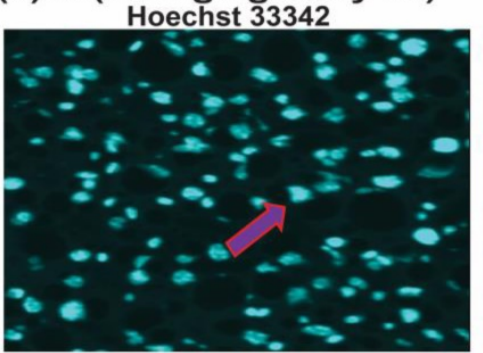

Fluorescein

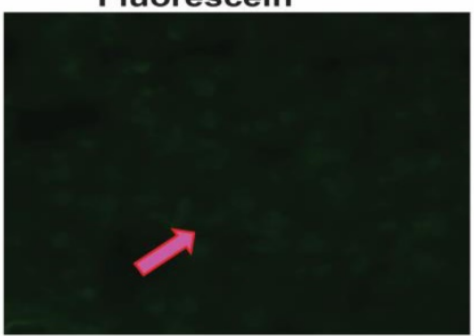

Fluorescein

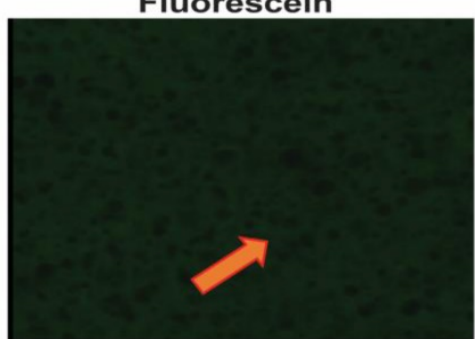

Fluorescein

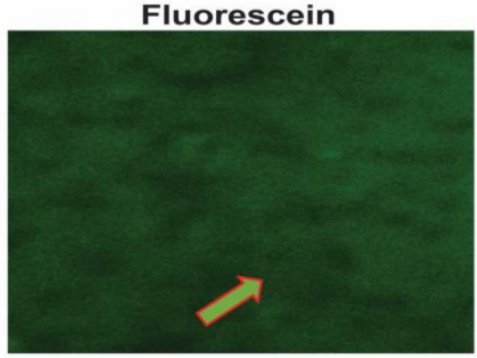

Fluorescein

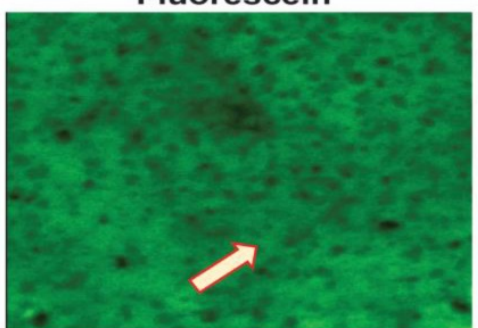

Fluorescein

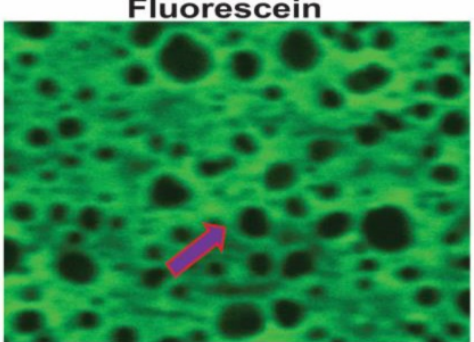

Merged

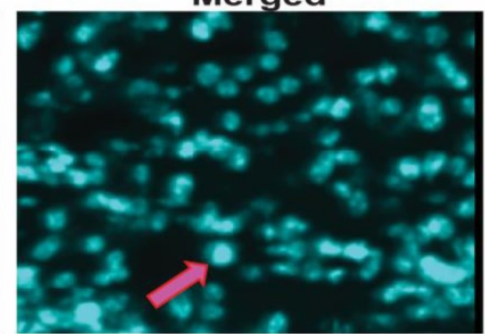

Merged

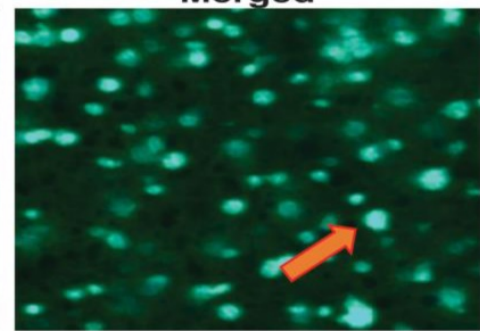

Merged

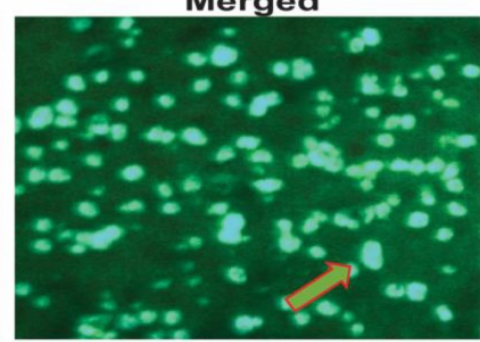

Merged

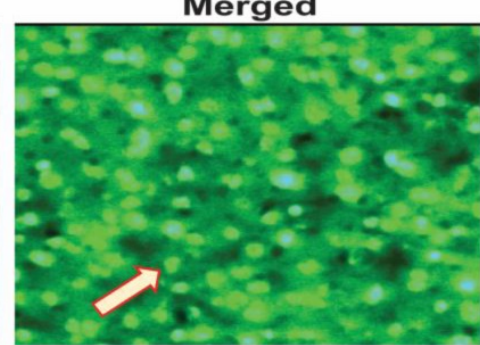

Merged

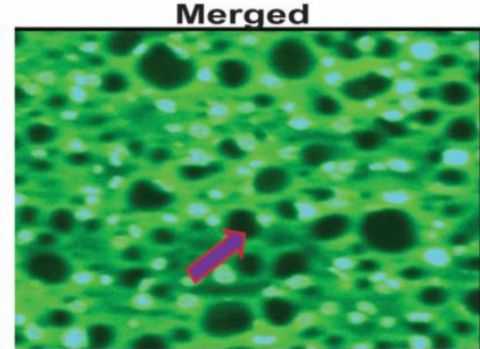

Figure 2. TX induces DNA damage in APL mice liver. Both control and TX-treated APL transgenic mice were sacrificed and the liver tissues were collected. The tissues were sectioned and sections fixed, and permeabilized, and DNA damage was analyzed by immunohistochemistry, tunnel assay, and fluorescence imaging. TX stimulated DNA damage in APL transgenic mice liver tissues (Fig.2[i-v]). 
[3A]

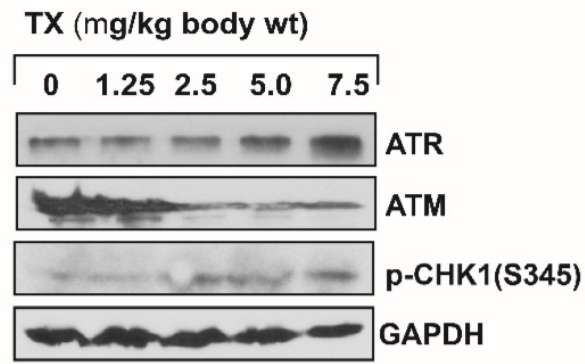

[3B]

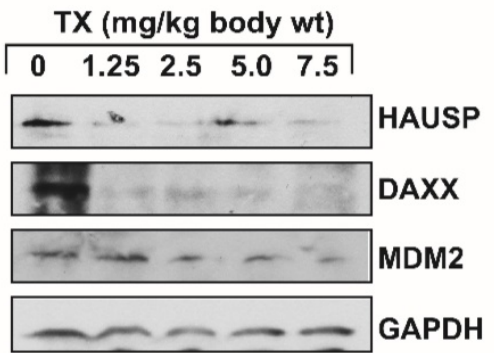

[3C]
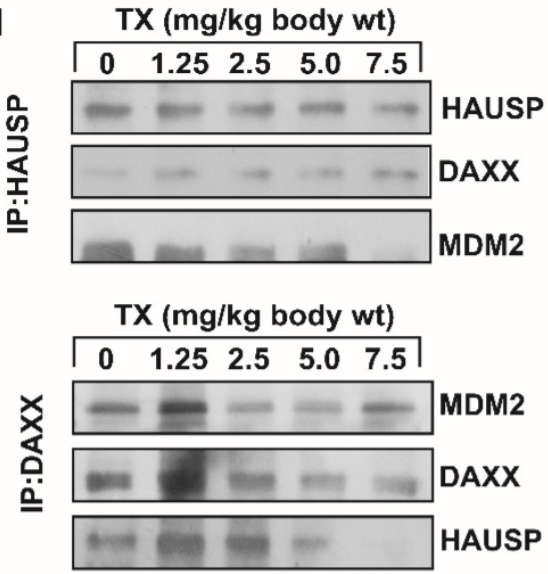

$\mathrm{TX}$ ( $\mathrm{mg} / \mathrm{kg}$ body $\mathrm{wt})$

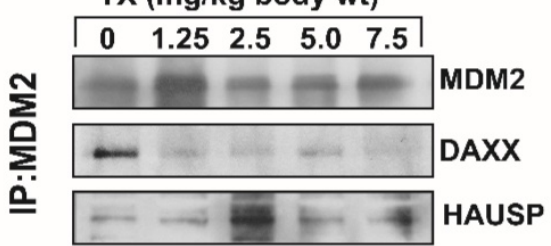

Figure 3. TX disrupts MDM2-DAXX-HAUSP complex. Both control and TX-treated APL transgenic mice were sacrificed and the liver tissues were collected in RIPA buffer. Protein lysates were made from all samples, and Western blotting was performed to assess the expression of complex molecules, protein kinases, and phosphorylation of CHK 1 at Ser 345 residue using both specific and phosphoactive antibodies. TX reduced the expression of complex molecules and ATM, and stimulated the expression of ATR through phosphorylation of CHK1 at Ser 345 residue in APL liver tissues (Fig.3A\&B). Immunoprecipitation (IP) was done using similar protein lysates of APL mice liver. It was found that TX also changed the association of complex molecules (Fig.3C).

\section{TX disrupts MDM2-DAXX-HAUSP complex}

Generally, DNA damage signal is transmitted by protein kinase [ATM \& ATR] and its downstream CHK1\& CHK2 residues phosphorylation leading to MDM2-DAXX-HAUSP complex disruption $(12,15)$. Our results showed that TX-induced DNA damage signal was transmitted through increased ATR and reduced ATM expression with stimulation of phosphorylation of CHK1 at serine residue (Fig.3A). It also disrupted MDM2-DAXX-HAUSP complex through reduced expression and changing association of complex molecules (Fig.3B\&C) in APL mice liver tissues.

\section{TX activates p53 in APL mice bone marrow cells}

It has been reported that DNA damage disrupts MDM2-DAXX-HAUSP complex leading to activation of p53 and MDM2 degradation in many cancer cells $(12,15,35)$. Our findings reveal that TX reduced MDM2 expression in a dose dependent fashion (Fig.4A\&B[i-v]), leading to p53 activation in APL mice bone marrow cells.

\section{TX activates p53 in APL mice liver tissues}

The results of our immunohistochemistry evaluation of APL mice liver tissue also show that TX down-regulated MDM2 expression in a dose dependent manner (Fig.5A\&B[i-v]), leading to p53 activation in APL mice bone marrow cells.

\section{TX modulates cell cycle regulation and apoptosis}

P53 is widely involved in cell cycle regulation and apoptosis in several cancers. We found that TX-induced p53 expression modulated cell cycle regulation through stimulation of $\mathrm{p} 21$, and reduction of cyclinD1, CDK2, CDK4, and CDK6 expression in APL mice liver tissues (Fig.6A). It also caused apoptosis in APL mice liver tissues through stimulation of caspase 3, and bax expression, and reduction of bcl-2 expression (Fig.6B).

\section{Discussion}

Trisenox (TX) has been used successfully for the treatment of all age groups of APL patients, either alone or in combination with ATRA; leading to a maximum efficacy and high survival rate. However, TX resistance has recently been reported in few APL patients $(5,6)$; underscoring the importance of searching for new targets of its action that may help to design new anti-leukemic drugs to cure APL patients more quickly and prevent growing resistance cases. Generally, TX enters into APL cells through diffusion and acts by various mechanisms in different pathways $(17,21,36,37)$. However, TX mode of action in mouse model of APL mostly remains elusive. We investigated a new mode of action of TX using a 
mouse model of APL. It has been reported that APL pathogenesis is caused by the formation of oncogene, PML-RARa $(28,30)$. We found that PML-RARa was expressed in our mice model of APL and TX reduced its expression in a dose-dependent fashion (Fig.1B[i-v]). TX also stimulated the formation of more promyelocytes with dense granules in APL mice bone marrow cells (Fig.1A). Previous studies have reported that TX induces DNA damage in APL cell lines $(20,21)$. Similarly, our finding also shows that TX caused genotoxicity in mice APL liver tissue(2B[i-v]). Accumulating evidence suggests that the DNA damage signal is transmitted by protein kinase (ATM\& ATR) and its residues phosphorylation leading to disruption of MDM2-DAXX-HAUSP complex and activation of p53 $(12,15,35)$. Our findings show that TX-induced genotoxic damage was transmitted by reduced ATM and stimulated ATR

[4A]

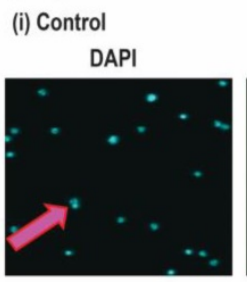

(ii) TX(1.25mg/kg body wt) DAPI

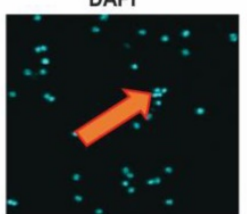

(iii) TX(2.5mg/kg body wt) DAPI

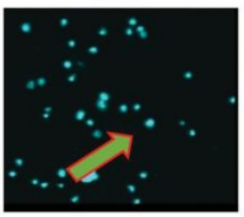

(iv) TX(5mg/kg body wt) DAPI

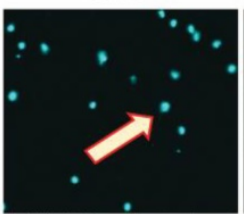

(v) TX(7.5mg/kg body wt) DAPI

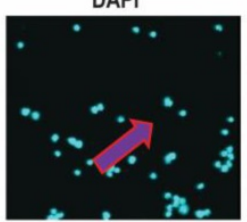

P53

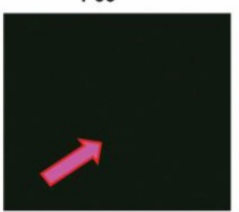

P53

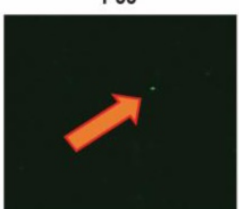

P53

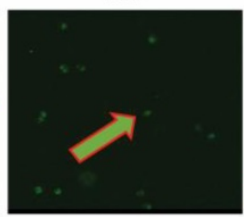

P53

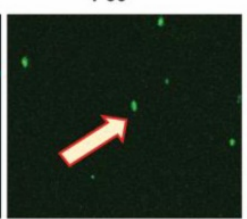

P53

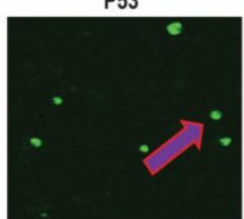

Merged

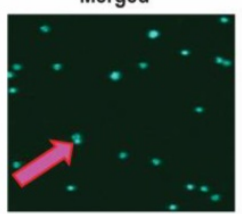

Merged

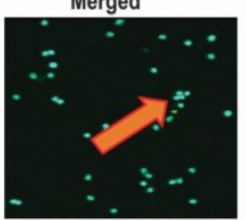

Merged

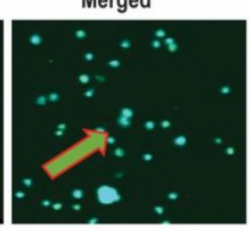

Merged

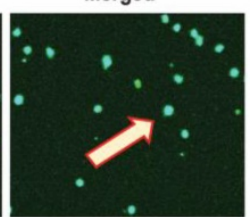

Merged

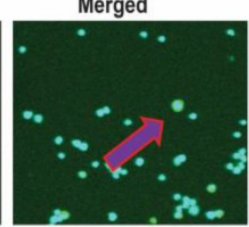

expression along with phosphorylation of CHK1 residue at ser 345 residue in mice APL liver tissue (Fig.3A). It also reduced the expression of MDM2-DAXX-HAUSP complex molecules (Fig.3B) and disrupted their association (Fig.3C) in APL mice liver tissue. TX-induced disruption of complex molecules led to activation of p53 and degradation of MDM2 in APL mice bone marrow cells (Fig.4A\&B[i-v]) as well as liver tissue (Fig.5A\&B[i-v]) in a dose-dependent fashion. It has been also reported that p53 is widely involved in cell cycle arrest and apoptosis in several cancers $(9,10)$. Our data show that TX-induced p53 expression was modulated cell cycle through activation of p21 and reduced expression of cyclin D1, CDK2, CDK4, and CDK6 in APL mice liver tissue (Fig.6A). TX also induced apoptosis through activation of caspase 3 and bax by reduced expression of bcl-2(Fig.6B).

[4B]

(i) Control

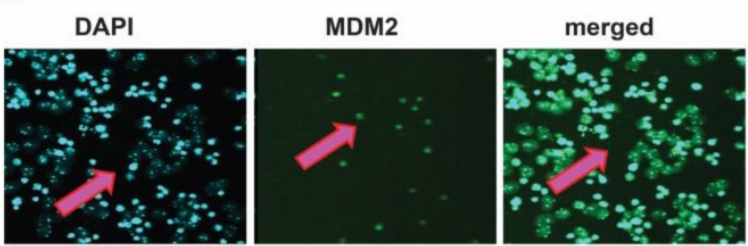

(ii) TX(1.25mg/kg body wt)

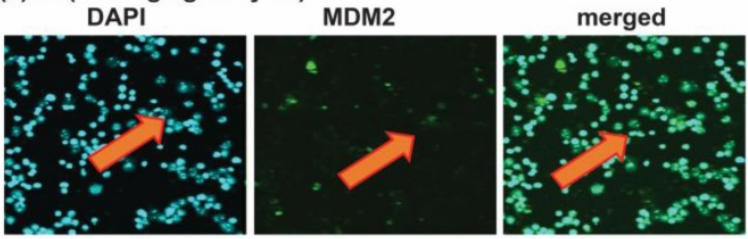

(iii) $\mathrm{TX}(2.5 \mathrm{mg} / \mathrm{kg}$ body wt)
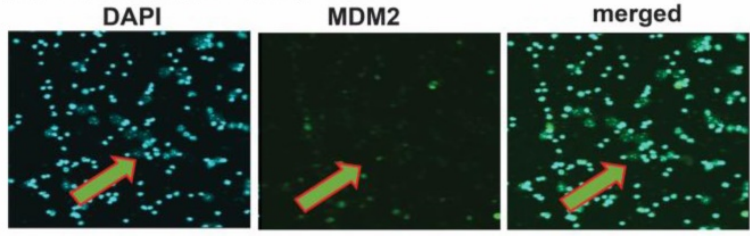

(iv) TX(5mg/kg body wt)

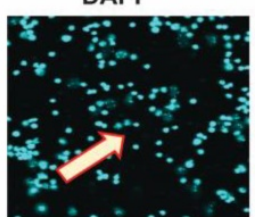

MDM2

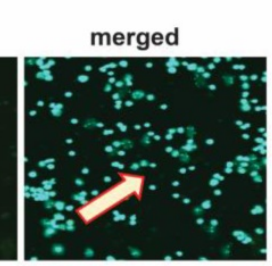

(v) TX(7.5mg/kg body wt)
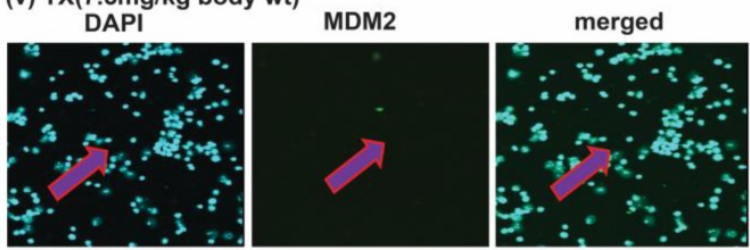

Figure 4. TX activates p53 in APL mice bone marrow cells. Immunocytochemistry was performed on both control and TX-treated APL transgenic mice bone marrow cells to check the expression levels of p53 and MDM2. TX stimulated p53 expression and reduced MDM2 expression in APL mice bone marrow cells (Fig.4A\&B). 


\section{[5A]}

(i) Control DAPI

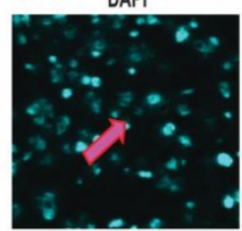

(ii) TX(1.25m $\mathrm{mg} / \mathrm{kg}$ body wt)

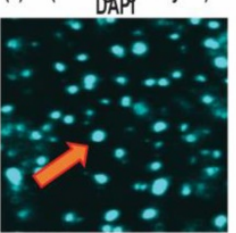

(iii) TX(2.5mg/kg body wt)

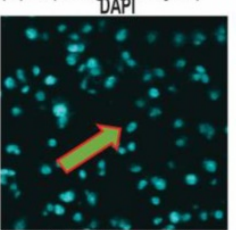

(iv) TX (5mg/kg body wt)

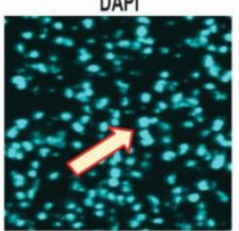

(v) TX (7.5mg/kg body wt)

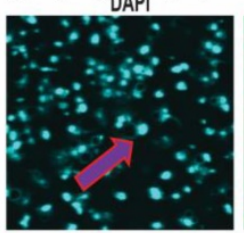

P53

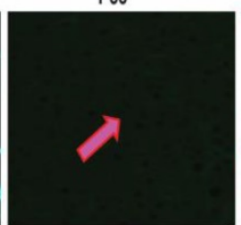

P53

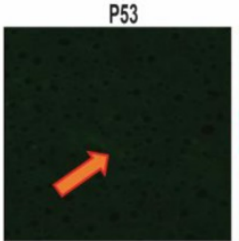

P53

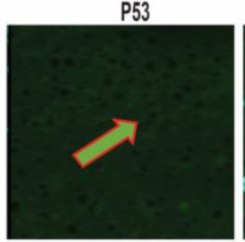

P53

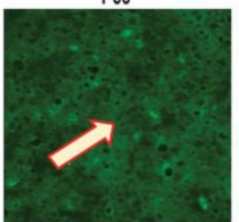

P53

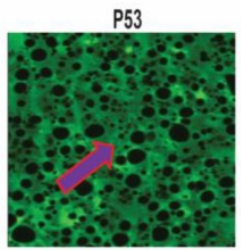

Merged
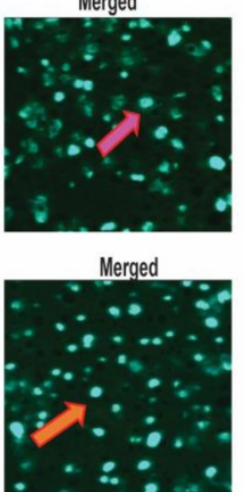

Merged

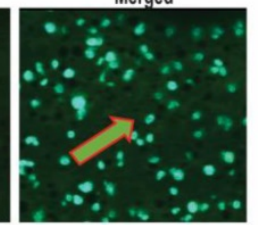

Merged

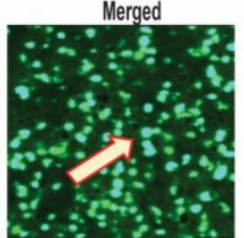

Merged

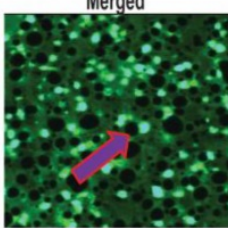

[5B]

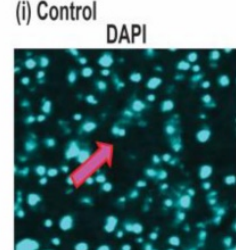

(ii) TX (1.25 $\mathrm{mg} / \mathrm{kg}$ body wt)

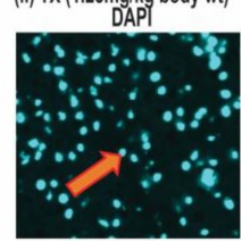

(iii) TX(2.5mg/kg body wt)

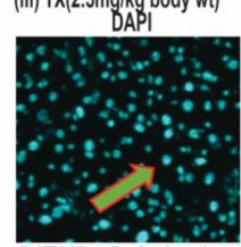

(iv)TX (5mg/kg body wt)

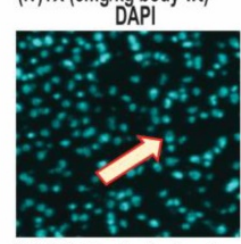

(v) TX (7.5mg/kg body wt)

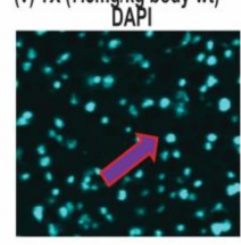

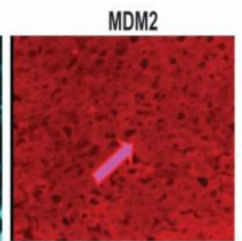

MDM2

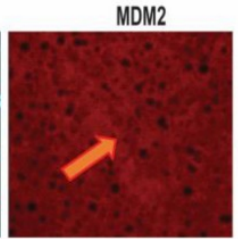

MDM2

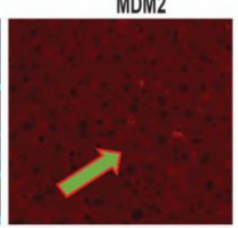

MDM2

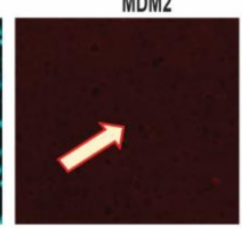

MDM2

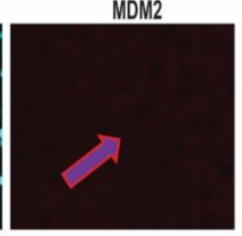

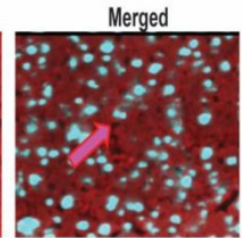

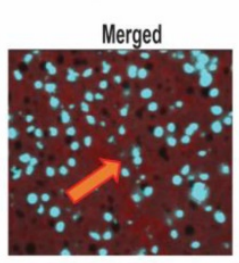

Merged

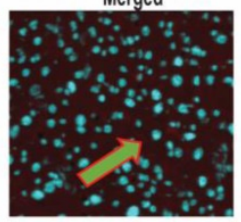

Merged
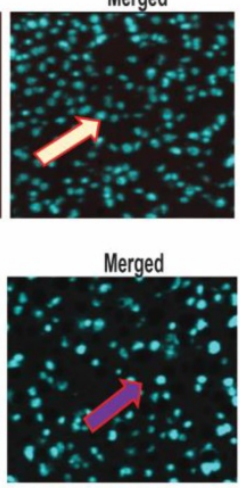

Figure 5. TX activates p53 in APL mice liver tissues. Immunohistochemistry (IHC) was performed on both control and TX-treated APL transgenic mice liver sections to check the expression levels of $\mathrm{p} 53$ and MDM2. TX activated p53 expression and reduced MDM2 expression in APL mice liver tissues sections (Fig.5A\&B).

In most of human cancers, p53 is mutated or remains functionally inactive by MDM2 and MDMX through E3 ligase activity. MDM2 is often involved in auto-degradation and proteasomal degradation inside cancer cells. Hence, it is always associated with DAXX and HAUSP to form MDM2-DAXX-HAUSP complex (15). Reactivation of p53 through disruption of complex and degradation of MDM2 could be an attractive and effective strategy for cancer therapy (38). The anti-cancer drugs, doxorubicin and VP-16 act inside cancer cells by successfully disrupting the MDM2-DAXX-HAUSP complex and degrading MDM2 expression (12). Trisenox inhibits the proliferation of APL cell lines through complex disruption, and MDM2 degradation leading to activation of p53. TX-induced p53 activation is involved in APL cell cycle modulation and apoptosis (35). TX-induced DNA damage signal transmission through protein kinase (ATM \&ATR) led to the disruption of complex molecules and change in their association, p53 activation, cell cycle regulation, and forcing APL mice liver and bone marrow cells to undergo apoptosis.

Inside APL cells, TX exerts its pharmacological effects through different molecular mechanisms that include reactive oxygen species [ROS] induction, oxidative stress, DNA damage, and p53 activation leading to cell cycle modulation and apoptosis $[8,17,21,39-41]$. P53, a tumor suppressor protein is activated in response to genotoxic and other stress-related factors, and is involved in cell cycle regulation and apoptosis of cancer cells (9.10). Generally, p53 expression is down-regulated in most cancer cells, predominantly through MDM2 and MDM4/MDMX by ubiquitination and proteasomal degradation. MDM2, a negative regulator of p53 normally associated with DAXX and HAUSP to form MDM2-DAXX-HAUSP complex [12]. The reactivation of p53 with anticancer drugs would be a promising strategy to cure cancer [38] by induction of cellular stress, MDM2 degradation, and self-ubiquitination $[12,15]$. 
[6A]

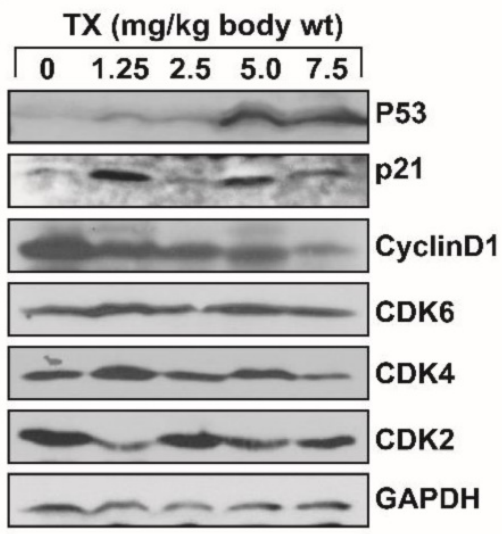

[6B]

\section{TX ( $\mathrm{mg} / \mathrm{kg}$ body wt)}

\begin{tabular}{|lllll|}
\hline 0 & 1.25 & 2.5 & 5.0 & 7.5
\end{tabular}

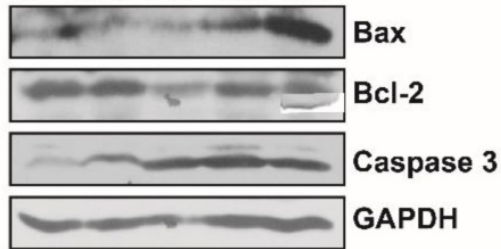

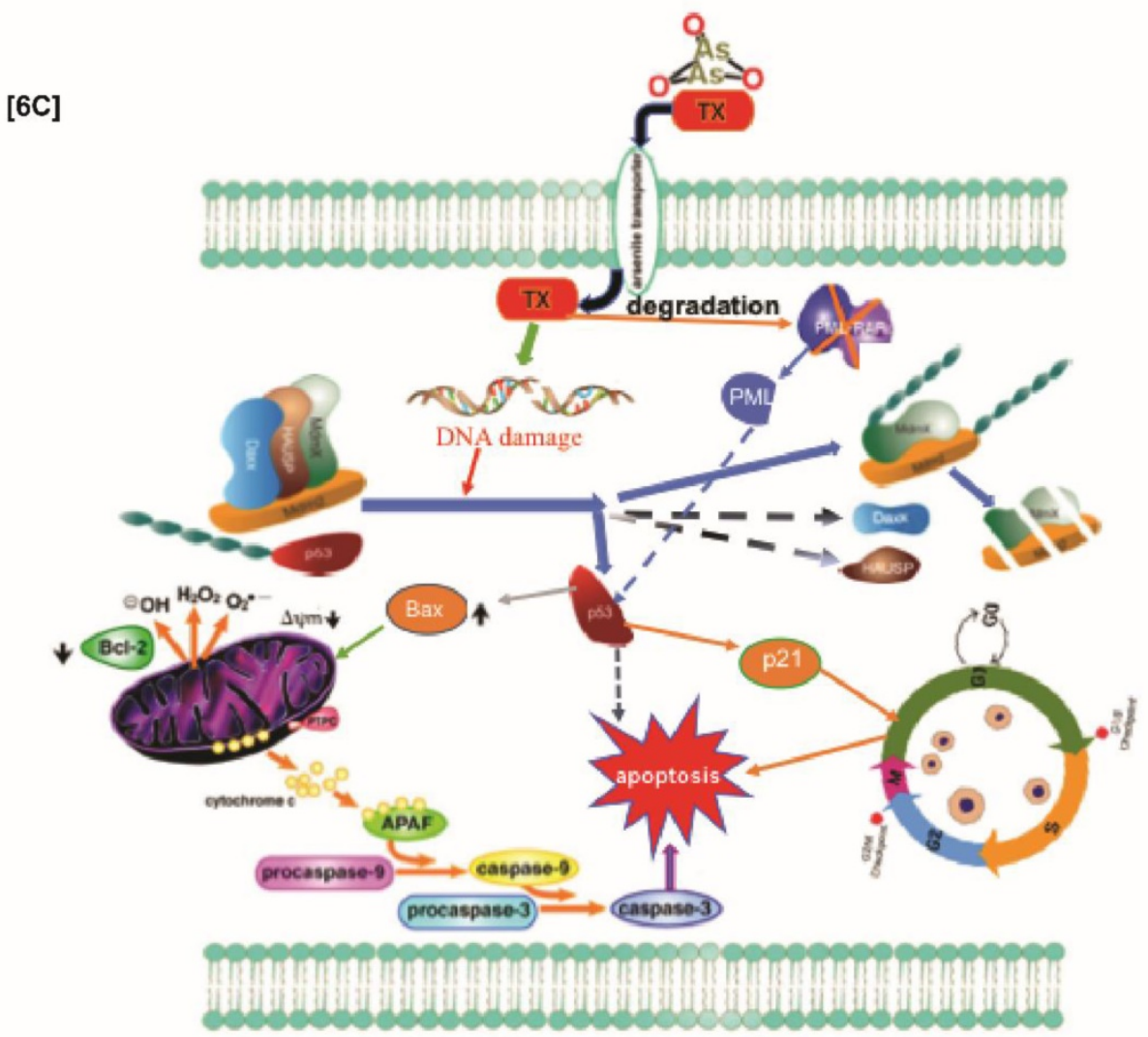

Figure 6. TX involves in cell cycle regulation and apoptosis. Both control and TX-treated APL transgenic mice were sacrificed and the liver tissues were collected in RIPA buffer. Protein lysates were made from all samples and Western blotting was performed to assess the expression levels of p53, p21, cyclin D1, CDK2, CDK4, CDK6, Bax, Bcl-2, and Caspase 3 using specific antibodies. TX significantly modulated cell cycle in APL mice liver tissues through activation of p53 and p21, and reduction of cyclin D1, CDK2, CDK4, CDK6 expression (Fig.6A). TX also induced apoptosis in APL transgenic mice liver tissue through stimulation of caspase 3 and Bax, and down-regulation of Bcl-2 expression (Fig.6B). In summary, TX-induced DNA damage signal was transmitted by protein kinases [ ATM \& ATR] leading to disruption of complex molecules and down-regulation of their expression. This process also led to the activation of $\mathrm{p} 53$ expression, cell cycle regulation, and apoptosis in APL tissues. TX also promoted more formation of promyelocytes with dense granules in APL mice bone marrow cells (Fig.6C).

It has been reported that ATO stimulates expression of p53 in resistant hepatocellular carcinoma cells (42). TX induces apoptosis in multiple myeloma and B-cell chronic lymphocytic leukemia cels through a p53-dependent signaling pathway $(43,44)$. Accumulating evidence reveals that TX prevents growth through accumulation of p53, p21, cell cycle arrest, and apoptosis in fibroblast cells, human gastric cancer cells, myeloma cells, lymphoid malignant cells, and acute promyelocytic leukemia (APL) cell lines. MDM2, a prominent antagonist of p53 regulates its expression in cancer cells and remains associated with DAXX and HAUSP to form MDM2-DAXX-HAUSP complex. This complex would be an important target of many anticancer drugs because its disruption leads to the activation of p53 (17-22). Promyelocytic leukemia (PML) gene is associated with p53 and is involved in pro-apoptotic 
events [26]. Promyelocytic leukemia zinc finger-retinoic acid receptor a (PLZF-RARa) stimulates cell proliferation in APL patients through a simultaneous down-regulation of the expression of both p53 and p21 proteins [27]. PML-transformation related protein (Trp53) is essential for the control of leukemia - initiating cells in mouse model of APL [28]. Pseudokinase Tribble 3 (TRIB3) promotes APL progression by inhibition of p53-mediated senescence. TX interacts with TRIB3/ PML-RARa and eradicates APL [29]. Our laboratory has previously found that TX disrupted MDM2-DAXX-HAUSP complex, reduced expression of MDM2, and activated p53 in APL cell lines [35]. However, there is no previous report evaluating it effect on MDM2-DAXX-HAUSP complex in a mouse model of APL. Hence, our findings are novel and correlate well with the current knowledge on the role of TX on the stimulation of p53 in cancer cells. For the first time, we hereby report that MDM2-DAXX-HAUSP complex is present in both liver tissues and bone marrow cells of transgenic APL mice. Most importantly, TX treatment down-regulates the expression of complex molecules and activates p53 as demonstrated in both western blotting and immunohistochemistry experiments simultaneously. This new mode of TX action in mice model of APL constitutes a novel target that may be very useful in designing new therapeutic strategies to overcome drug resistance in APL patients.

\section{Acknowledgements}

We are thankful to Dr. Ibrahim Farah, professor and manager of Animal Core facility, Department of Biology, Jackson State University for continuous support and advice for maintaining and breeding APL transgenic mice in the laboratory.

\section{Grant Support}

This research was financially supported by National Institutes of Health NIMHD Grant No. G12MD007581, through the RCMI-Center for Environmental Health at Jackson State University, Jackson, MS, USA.

\section{Authors Contributions}

Conception and Design: Sanjay Kumar and Paul B. Tchounwou. Analysis and interpretation of data: Sanjay Kumar. Writing, review and revision of the manuscript: Sanjay Kumar and Paul B. Tchounwou.

\section{Competing Interests}

The authors have declared that no competing interest exists.

\section{References}

1. Powell BL. Arsenic trioxide in acute promyelocytic leukemia: potion not poison. Expert Rev. Anticancer Ther 2011; 11: 1317-9.

2. Kumar S, Tchounwou PB. Molecular mechanisms of cisplatin cytotoxicity in acute promyelocytic leukemia cells. Oncotarget 2015; 6: 40734-6.

3. Grignani F, Fagioli M, Alcalay M. Acute promyelocytic leukemia: from genetics to treatment. Blood 1994; 83: 10-25.

4. Lo-Coco F, Avvisati G, Vignetti M, et al. Retinoic acid and arsenic trioxide for acute promyelocytic leukemia. N Engl J Med 2013; 369:111-21.

5. Tomita A, Kiyoi H, Naoe T. Mechanisms of action and resistance to all-trans retinoic acid (ATRA) and arsenic trioxide (As2O3) in acute promyelocytic leukemia. Int. J. Hematol . 2013; 97:717-25.

6. Lou Y, Ma Y, Sun J, et al. Evaluating frequency of PML-RARA mutations and conferring resistance to arsenic trioxide-based therapy in relapsed acute promyelocytic leukemia patients. Ann Hematol. 2015; 94:1829-37.

7. Baresova P, Musilova J, Pitha PM, et al. p53 tumor suppressor protein stability and transcriptional activity are targeted by Kaposi'ssarcoma-associated herpesvirus encoded viral interferon regulatory factor 3. Mol Cell Biol. 2014; 34:386-99.

8. Yedjou CG, Tchounwou PB. Modulation of p53, c-fos, RARE, cyclin A, and cyclin D1 expression in human leukemia (HL-60) cells exposed to arsenic trioxide. Mol Cell Biochem. 2009;331:207-14.

9. Vogelstein B, Lane D, Levine AJ. Surfing the p53 network. Nature 2000; 408:307-10.

10. Vousden $\mathrm{KH}, \mathrm{Lu} X$. Live or let die: the cell's response to p53. Nat Rev Cancer 2002; 2:594-04

11. Kruse JP, Gu W. Modes of p53 regulation. Cell. 2009;137:609-22.

12. Zhang X, Gu L, Li J, et al. Degradation of MDM2 by the interaction between berberine and DAXX leads to potent apoptosis in MDM2-overexpressing cancer cells. Cancer Res 2010; 70:9895-04.

13. Maltzman W, Czyzyk L. UV irradiation stimulates levels of p53 cellular tumor antigen in nontransformed mouse cells. Mol. Cell Biol.1984; 4:1689-94.

14. Kastan MB, Onyekwere O, Sidransky D, et al. Participation of p53 protein in the cellular response to DNA damage. Cancer Res. 1991;51:6304-11

15. Meek DW.Tumour suppression by p53: a role for the DNA damage response? Nat Rev Cancer 2009; 9:714-23.

16. Tang J, Qu LK, Zhang J, et al. Critical role for Daxx in regulating MDM-2. Nat Cell Biol 2006; 8:855-62.

17. Miller WH Jr, Schipper HM, Lee JS, et al. Mechanisms of action of arsenic trioxide. Cancer Res. 2002; 62:3893-03.

18. Jiang $\mathrm{XH}$, Wong $\mathrm{BC}$, Yuen $\mathrm{ST}$, et al. Arsenic trioxide induces apoptosis in human gastric cancer cells through up-regulation of p53 and activation of caspase-3. Int J Cancer. 2001:91:173-9.

19. Park WH, Seol JG, Kim ES, et al. Arsenic trioxide-mediated growth inhibition in MC/CAR myeloma cells via cell cycle arrest in association with induction of cyclin-dependent kinase inhibitor, p21, and apoptosis. Cancer Res 2000; 60: 3065-71.

20. Yedjou C, Tchounwou $P$, Jenkins J, et al. Basic mechanisms of arsenic trioxide (ATO)-induced apoptosis in human leukemia (HL-60) cells. J Hematol Oncol 2010; 3:28-35.

21. Kumar S, Yedjou CG, Tchounwou PB. Arsenic trioxide induces oxidative stress, DNA damage, and mitochondrial pathway of apoptosis in human leukemia (HL-60) cells. J Exp Clin Cancer Res. 2014; 33:42.

22. Zhang W, Ohnishi $\mathrm{K}$, Shigeno $\mathrm{K}$, et al. The induction of apoptosis and cell cycle arrest by arsenic trioxide in lymphoid neoplasms. Leukemia 1998; 12: 1383 - 91.

23. Ma DC, Sun $\mathrm{YH}$, Chang KZ, et al. Selective induction of apoptosis of NB4 cells from G2 + M phase by sodium arsenite at lower doses. Eur J Haematol 1998; 61: 27-35.

24. Torii S, Egan DA, Evans RA, et al. Human Daxx regulates Fas-induced apoptosis from nuclear PML oncogenic domains (PODs). EMBO J 1999; 18:6037- 49 .

25. Song MS, Song SJ, Kim SY, et al. The tumour suppressor RASSF1A promotes MDM-2 self-ubiquitination by disrupting the MDM2-DAXX-HAUSP complex. EMBO J 2008; 27:1863-74

26. Guo A, Salomoni P, Luo J, et al. The function of PML in p53-dependent apoptosis. Nat. Cell Biol 2000; $2: 730-6$.

27. Choi WI, Yoon JH, Kim MY, et al. Promyelocytic leukemia zinc finger-retinoic acid receptor a (PLZF-RARa), an oncogenic transcriptional repressor of cyclin-dependent kinase inhibitor 1A (p21WAF/CDKN1A) and tumor protein p53 (TP53) genes. J Biol Chem. 2014; 289: 18641-56.

28. Ablain J, Rice K, Soilihi $\mathrm{H}$, et al.. Activation of a promyelocytic leukemia-tumor protein 53 axis underlies acute promyelocytic leukemia cure. Nat. Med $2014 ; 20: 167-74$

29. Li K, Wang F, Cao WB, et al. TRIB3 Promotes APL Progression through Stabilization of the Oncoprotein PML-RARa and Inhibition of p53-Mediated Senescence. Cancer Cell. 2017; 31:697-10.

30. Rego EM, He LZ, Warrel RP Jr, et al. Retinoic acid (RA) and As2O3 treatment in transgenic models of acute promyelocytic leukemia (APL) unravel the distinct nature of the leukemogenic process induced by the PML-RARalpha and PLZF-RARalpha oncoproteins. Proc Natl Acad Sci 2000: 97 :10173-8.

31. Madaan A, Verma R, Singh AT, et al. A stepwise procedure for isolation of murine bone marrow and generation of dendritic cells. J Biol Methods 2014; $1: e 1$. 
32. Tallman MS, Altman JK. How I treat acute promyelocytic leukemia. Blood 2009; 114:5126-35.

33. Guha M, Kumar S, Choubey V, et al. Apoptosis in liver during malaria: role of oxidative stress and implication of mitochondrial pathway. FASEB J. 2006; 20:1224-6.

34. Singh NK, Quyen DV, Kundumani-Sridharan V, et al. AP-1 (Fra-1/c-Jun)-mediated induction of expression of matrix metalloproteinase-2 is required for 15S-hydroxyeicosatetraenoic acid-induced angiogenesis. AP-1 (Fra-1/c-Jun)-mediated induction of expression of matrix metalloproteinase-2 is required for 15S-hydroxyeicosatetraenoic acid-induced angiogenesis. J Biol Chem. 2010; 285:16830-43.

35. Kumar S, Brown A, Tchounwou PB. Trisenox disrupts MDM2-DAXX-HAUSP complex and activates p53, cell cycle regulation and apoptosis in acute leukemia cells. Oncotarget 2018; 9:33138- 48.

36. Lengfelder E, Hofmann WK, Nowak D. Impact of arsenic trioxide in the treatment of acute promyelocytic leukemia. Leukemia 2012; 26:433-42.

37. Emadi A, Gore SD. Arsenic trioxide - An old drug rediscovered. Blood Rev. 2010; 24: 191-9.

38. Wade M, Li YC, Wahl GM. MDM2, MDMX and p53 in oncogenesis and cancer therapy. Nat Rev Cancer 2013; 13: 83-96.

39. Coombs CC, Tavakkoli M, Tallman MS. Acute promyelocytic leukemia: where did we start, where are we now, and the future. Blood Cancer J. $2015 ; 5: \mathrm{e} 304$.

40. Sanz MA, Lo-Coco F. Modern approaches to treating acute promyelocytic leukemia. J Clin Oncol. 2011;29:495-503.

41. Zhou GB, Zhang J, Wang ZY, Chen SJ, Chen Z. Treatment of acute promyelocytic leukaemia with all-trans retinoic acid and arsenic trioxide: a paradigm of synergistic molecular targeting therapy. Philos Trans R Soc Lond B Biol Sci. 2007; 362:959-71.

42. Zheng T, Yin D, Lu Z, et al. Nutlin-3 overcomes arsenic trioxide resistance and tumor metastasis mediated by mutant p53 in Hepatocellular Carcinoma. Mol Cancer. 2014;13:133.

43. Kircelli F, Akay C, Gazitt Y. Arsenic trioxide induces p53-dependent apoptotic signals in myeloma cells with SiRNA-silenced p53: MAP kinase pathway is preferentially activated in cells expressing inactivated p53. Int J Oncol. 2007; 30:993-01.

44. Zhang XH, Feng R, Lv M, et al. Arsenic trioxide induces apoptosis in B-cell chronic lymphocytic leukemic cells through down-regulation of survivin via the p53-dependent signaling pathway. Leuk Res. 2013; 37:1719-25. 